\title{
Promoting Protective Scale Formation at Lower Temperatures via Surface Finishing: Effects on the Establishment, Structure, and Chemistry in Haynes 214 High- Temperature Oxidation-Resistant Nickel Alloy
}

Stephen House ${ }^{1}$, Henry Ayoola ${ }^{2}$, John Lyons ${ }^{3}$, Bingtao $\mathrm{Li}^{4}$, Judith Yang ${ }^{2}$ and Brian Gleeson ${ }^{2}$

${ }^{1}$ University of Pittsburgh, Pittsburgh, Pennsylvania, United States, ${ }^{2}$ University of Pittsburgh, United States, ${ }^{3}$ Westinghouse Electric Company, United States, ${ }^{4}$ Haynes International, United States

Haynes® $214 \circledR$ is a Ni-Cr-Al-Fe alloy widely employed for its excellent resistance to oxidation and corrosion in high-temperature oxidizing environments, such as direct flame impingement, burner assemblies, and catalytic converter internals of automobiles. The resistance is attributable to the formation of a protective and adherent aluminum oxide scale. The oxidation behavior of 214 , however, is temperature-dependent. Alumina-scale formation on 214 is kinetically favorable at exposure temperatures above $955^{\circ} \mathrm{C}$; below this temperature a less-protective scale containing nickel oxide is apt to form. This could be problematic for lower-temperature applications, particularly those involving multiple oxidants.

Surface finishing of metallic parts and structures is often done for aesthetic or practical reasons. For example, it has been shown that the surface treatment can impact the corrosion resistance of metals.[1,2] More recently in a study of $\mathrm{Ni}-\mathrm{Al}$ and $\mathrm{Ni}-\mathrm{Al}-\mathrm{Cr}$ alloys at $1100{ }^{\circ} \mathrm{C}$, surface abrasion was used to directly promote $\mathrm{Al}_{2} \mathrm{O}_{3}$-scale establishment.[3] Inspired by this, we investigated whether surface modification via abrasion could be used to facilitate $\mathrm{Al}_{2} \mathrm{O}_{3}$-scale formation on 214 alloys at lower temperatures. This presentation discusses our study of the impact of typical surface finishes on the alloy structure and the associated response to short-term oxidation exposures.

In the current study, three surface treatments were investigated: polishing with graded $\mathrm{SiC}$ sandpapers finishing at 500- or 1200-grit, and vapor blasting (VB) with 300 mesh silica beads. The surface-finished samples were then oxidized in air at $800{ }^{\circ} \mathrm{C}$ for 2 hours. Surface and topographical characterization was performed using atomic force microscopy (AFM) and scanning electron microscopy (SEM), while focused ion beam (FIB-SEM) cross-sectional lift-outs were examined using scanning transmission electron microscopy (STEM), energy-dispersive X-ray spectroscopy (EDXS), and ASTAR nanoscale orientation mapping to characterize the subsurface. This examination revealed that surface finishing does indeed impact the oxide scale formation on 214 down to the nano-scale, and provide insight into the relative importance of various structural aspects.

The oxidation process did not appear to meaningfully alter the surface topography and roughness of the three samples. Moreover, and despite quite different post-finishing deformation structures in both form and extent, the three samples exhibited some surprisingly similar recrystallization structures after oxidation (Figure 1). The discrete layer of heavily deformed grains in the 500-grit, the half-as-thick layer in the 1200-grit, and the deep gradient of deformed grains extending at least ten times as deep in the VB all yielded a discrete recrystallization zone (RZ) with depths of similar sub-micron magnitude at the surface. The grains below the upper RZ (URZ) in the VB also recrystallized into an extensive, yet much finer-grained, lower RZ, while the grit-polished samples' bulk (the grains that did not experience deformation from the surface treatment) retained their large grain sizes. This apparent disconnect between RZ (URZ, in VB) depth and initial deformation depth is notable and surprising, suggesting an additional effect (e.g., scaling-induced strain) or kinetic limitation that is influencing the recrystallization of this near-surface layer. 
The two grit-polishing treatments also did not exhibit a meaningful difference in the scale produced. A continuous, conformal oxide scale, without any noticeable voids or pinholes, spanned the entire length of the FIB lamella atop the RZ. The thickness was very uniform and effectively identical in both grit-polished samples. The composition of the scale was more complex, being a tri-layer laminatetype scale of alumina with a continuous layer of chromia running through the middle. (Figure 2a-b) This, along with the residual $\mathrm{Ni}$ on the surface, suggests the chromia band corresponds to the original sample surface, with alumina growth both inward and outward. In the VB sample, the oxide scale is still conformal, but $30 \%$ thicker and over twice as rough. It still contained chromia inclusions, but of a discontinuous "mountain-like" nature. These measurements found no correlation between the oxide thickness, RMS surface roughness, and initial deformation depth.

The apparent - though slight - correlation between (U)RZ depth and oxide scale thickness suggests that these zones play an important role in controlling $\mathrm{Al}_{2} \mathrm{O}_{3}$-scale establishment. This is supported by comparing the elemental composition profiles across the various regions (Figure 2c-d), wherein an abrupt and uniform depletion of $\mathrm{Al}$ occurred at the (U)RZ boundary. This indicates that the (U)RZ is essentially the sole source of $\mathrm{Al}$ for the scale, and the flat profile indicates a high diffusivity of $\mathrm{Al}$ in these zones.

(U)RZ grain analysis revealed different size distributions for all three samples, meaning - if parabolic oxide growth kinetics are assumed - the scale thickness cannot solely be accounted for by subsurface grain size. This is supported by the Al depletion being restricted to the (U)RZ even in the VB case, despite the LRZ grains being over ten times smaller, and thus would have been expected to have a much higher Al diffusivity. Thus, scale establishment is clearly dictated by the (U)RZ, but not necessarily in the expected manner. Additional experiments to determine any difference in the nature of the grain boundaries (misorientation, etc.) and the presence of strain via nanoscale orientation mapping are underway. These studies will hopefully also shed light on the kinetic origin of the interesting two-zone structure of the VB.[4]

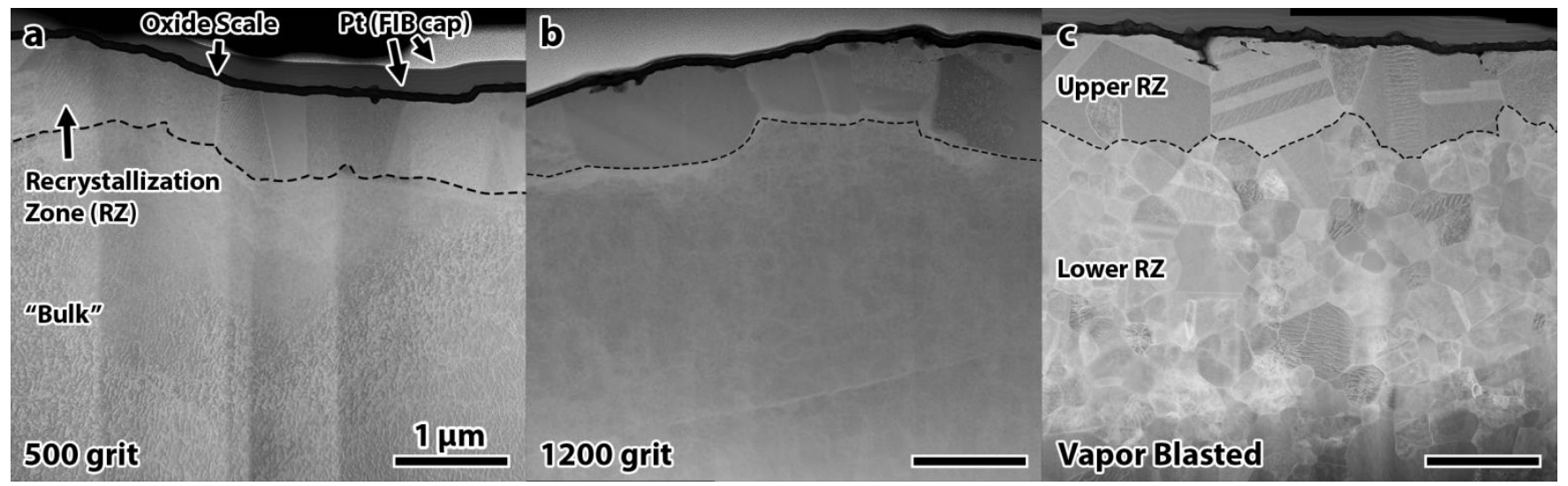

Figure 1. Representative portions from HAADF-STEM micrographs of cross-sectional FIB lamella taken from post-oxidation specimens of (a) 500-grit polished, (b) 1200-grit polished, and (c) VB Haynes 214 alloy. 

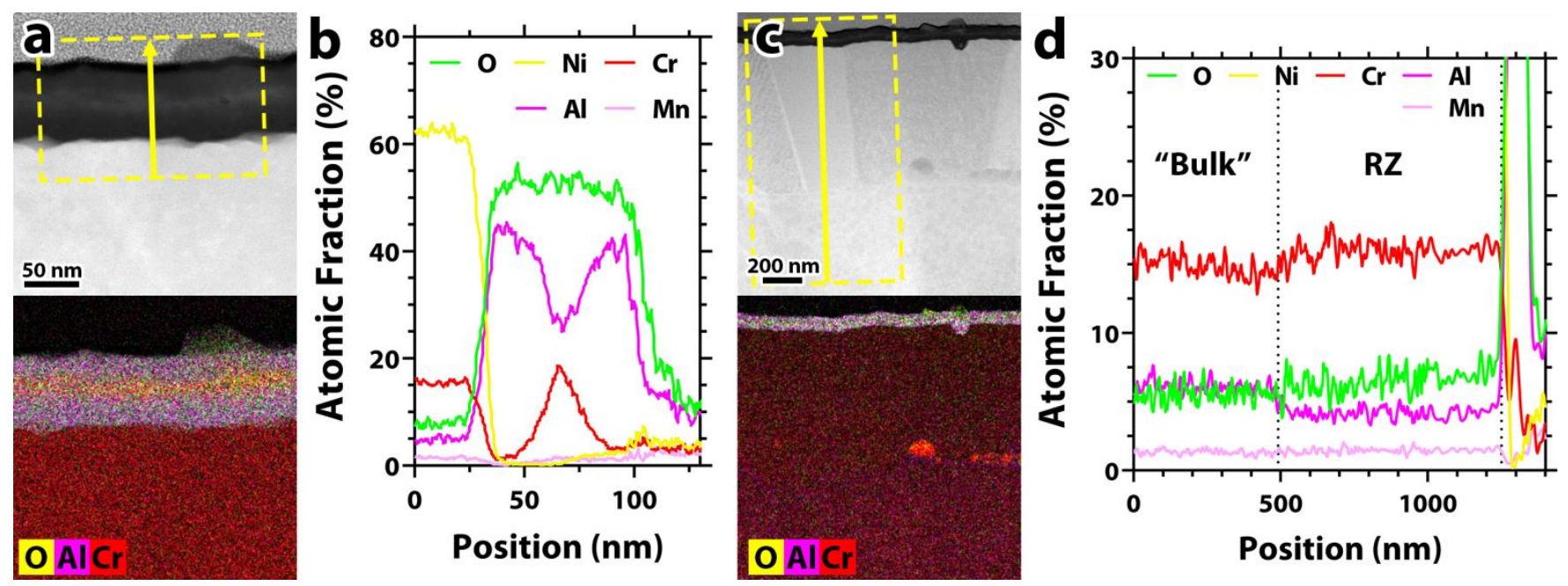

Figure 2. (a) HAADF-STEM image and EDS map of a representative section of the oxide scale in the 500 grit sample. (b) The corresponding EDS line profile of the dashed box in (a). (c) HAADF-STEM image and EDS map of a representative section of the 500-grit sample spanning the scale, recrystallization zone (RZ), and bulk. (d) The corresponding EDS line profile of the dashed box in (c).

\section{References}

1. X.B. Chen, C. Ke, N. Birbilis, Magnesium Technology 2014: pp.319-329 (2014)

2. C.O. Hoog, N. Birbilis, M.X. Zhang, Y. Estrin, Key Engineering Materials, 384: pp.229-240 (2008)

3. Y. Kang, PhD Dissertation, University of Pittsburgh (2016)

4. We acknowledge funding from the US National Science Foundation [CHE-1534630, DMR-1508417, CMMI-1905647]. This research used resources of the Environmental TEM Catalysis Consortium (ECC), which is supported by the University of Pittsburgh and Hitachi High Technologies. 